\title{
Supporting Discovery in Medicine by Association Rule Mining of Bibliographic Databases
}

\author{
Dimitar Hristovski ${ }^{1}$, Sašo Džeroski ${ }^{2}$, Borut Peterlin ${ }^{3}$, and Anamarija Rožić- \\ Hristovski $^{4}$ \\ 'IBMI, Medical Faculty; Vrazov trg 2/2, 1105 Ljubljana, Slovenia \\ dimitar.hristovski@mf.uni-lj.si \\ ${ }^{2}$ Institute Jozef Stefan; Jamova 39, 1000 Ljubljana, Slovenia \\ saso.dzeroskilijs.si \\ ${ }^{3}$ Department of Human Genetics, Clinical Center Ljubljana; Zaloska, 1000 Ljubljana, \\ Slovenia

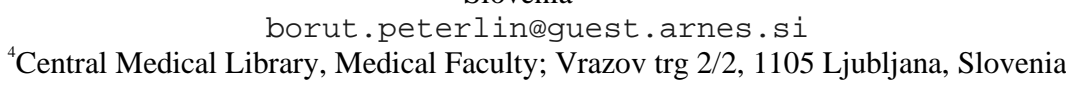 \\ anamarijadibmi.mf.uni-lj.si
}

\begin{abstract}
The paper presents an interactive discovery support system for the field of medicine. The intended users of the system are medical researchers. The goal of the system is: for a given starting concept of interest, discover new, potentially meaningful relations with other concepts that have not been published in the medical literature before. We performed two types of preliminary evaluation of the system: 1) by a medical doctor and 2) by automatic means. The preliminary evaluation showed that our approach for supporting discovery in medicine is promising, but also that some further work is needed, especially on limiting the number of potential discoveries the system generates.
\end{abstract}

\section{Introduction}

The two main questions addressed by this paper are: 1. Is it possible to discover new, potentially meaningful relations between medical concepts by searching and analysing the documents from a bibliographic database such as Medline? and 2. To what degree can be the discovery process automated? As an attempt to deal with these issues we developed an interactive discovery support system based on association rule mining of the Medline bibliographic database. Its intended use is as a generator for research ideas that should be then investigated by traditional medical methods.

The idea of discovering new relations from a bibliographic database was introduced by Swanson [1,2] who managed to make seven medical discoveries that have been published in relevant medical journals $[1,3]$.

Our system is based on Swanson's ideas, but there are however, several notable differences between our approach and theirs. Instead of using title words as a representation of the Medline documents' meaning, we use the MeSH descriptors. The MeSH descriptors are assigned to documents by human experts during the document indexing stage. We believe that the MeSH descriptors represent more precisely what a particular document is about. We use association rules as a measure of relationship between medical concepts while Swanson uses word frequencies. We have built a 
large association rule base by pre-calculating and storing the association rules in a database management system. This allows us to build a truly interactive discovery support system with fast response.

\section{Background}

Swanson's work. Swanson's discovery support process is based on the concepts of complementary literatures and noninteractive literatures [1,2]. If one set of articles (XY) reports an interesting relation between concepts $\mathrm{X}$ and $\mathrm{Y}$, and a different set of articles (YZ) reports a relation between $\mathrm{Y}$ and Z, but nothing has been published concerning a possible link between $\mathrm{X}$ and $\mathrm{Z}$, then $\mathrm{XY}$ and $\mathrm{YZ}$ are called complementary literatures. Generally, $\mathrm{XY}$ and $\mathrm{YZ}$ are complementary if a potentially new relation can be inferred by considering them together that can not be inferred from either of them separately. For example, $\mathrm{X}$ might be a disease, $\mathrm{Y}$ a physiological function associated with $\mathrm{X}$ and $\mathrm{Z}$ a substance or drug which induces or regulates the physiological function $Y$. If the readers and authors of one literature are not acquainted with the other, and vice versa, as might often be the case with two different specialties, then the two literatures are noninteractive. By combining the concepts of complementary and noninteractive literatures, Swanson developed the concept of undiscovered public knowledge meaning that although the literatures XY and YZ represent publicly available knowledge, the potentially new relation between $\mathrm{X}$ and $\mathrm{Z}$ remains undiscovered and is a valuable source of new discoveries.

In the beginning, Swanson performed the discovery process manually by searching the Medline database [2]. Later he added software support for some of the stages of the process. His current system is called ARROWSMITH and is described in detail in [3].

Medline. The Medline database is a product of the US National Library of Medicine (NML). Because of its coverage and free accessibility, Medline is the most important bibliographic database in the field of biomedicine. Each citation is associated with a set of MeSH (Medical Subject Headings) terms that describe the content of the item [4].

Medical Subject Headings (MeSH). MeSH comprises NLM's controlled vocabulary and thesaurus used for indexing articles and for searching MeSH-indexed databases, including Medline [4].

Unified Medical Language System (UMLS). The Unified Medical Language System (UMLS) project that NLM began in 1986 was undertaken with the goal of providing a mechanism for linking diverse medical vocabularies as well as sources of information. UMLS consists of three components: the Metathesaurus, Specialist Lexicon and Semantic Network [4,5].

\section{System Description}

Goal and Basic Premises. The system we developed is an interactive discovery support system for the field of medicine and is supposed to be used as a generator of 
new, potentially meaningful relations between a starting known concept of interest and other concepts.

The Medline database is used heavily by medical researchers. Traditionally it is used to check what is new in the literature on a particular topic of interest or to check if a medical discovery has already been published. In contrast to the traditional use of Medline, our system actively helps in the discovery process by generating potentially new discoveries and research ideas by analyzing the Medline database.

We used the major MeSH descriptors assigned to a Medline record as a representation of the contents of the article the record is about. Some of the MeSH descriptors are designated as major (preceded by an asterisk in the Medline record). Major descriptors are those that are the main topic of the article.

We used association rules [6] between pairs of medical concepts as a method to determine which concepts are related to a given starting concept. In our system an association rule of the form

$X->Y$ (confidence, support)

means that in confidence percent of articles containing $\mathrm{X}, \mathrm{Y}$ is present and that there are support number such articles. In other words, we take concept co-occurrence as an indication of a relation between concepts. If $\mathrm{X}$ is a disease, for example, then some possible relations might be: has-symptom, is-caused-by, is-treated-with-drug and so on. We do not try to find out the kind of relation. This can not be done by using the $\mathrm{MeSH}$ descriptors assigned to an article because there is no information about the relation between the descriptors.

Algorithm. We calculated all the associations between the major $\mathrm{MeSH}$ descriptors. We did this regardless of the confidence and support values and for two Medline time segments: 1990-1995 and 1996-1999. The calculated associations are stored in a database management system: there are currently more than 11.000 .000 associations in the rule base. The calculation of the association rules was much simplified by the use of the data contained in the UMLS.

The large association rule base is a foundation upon which the algorithm for discovering new relations between concepts proceeds as described in Table 1 . The user of the system should then evaluate the proposed $(X, Z)$ pairs and select among them those that deserve further investigation.

Table 1. The algorithm for discovering new relations between medical concepts.

1. Let $\mathrm{X}$ be a given starting concept of interest.

2. Find all concepts $\mathrm{Y}$ such that there is an association rule $\mathrm{X} \rightarrow \mathrm{Y}$.

3 . Find all concepts $\mathrm{Z}$ such that there is an association rule $\mathrm{Y} \rightarrow \mathrm{Z}$.

4. Eliminate those $Z$ for which an association $X->Z$ already exists.

5. The remaining $Z$ concepts are candidates for a new relation between $X$ and $Z$.

Because in Medline each concept can be associated with many other concepts, the possible number of $\mathrm{X} \rightarrow \mathrm{Z}$ combinations can be extremely large. In order to deal with this combinatorial problem, the algorithm incorporates filtering (limiting) and ordering capabilities. The default filtering that can not be relaxed is that only the associations between major MeSH headings are considered by the system. The related concepts can be limited by the semantic type to which they belong. Each MeSH descriptor belongs to one or more semantic types. For example, if the starting concept $\mathrm{X}$ is a disease (semantic type disease or syndrome) then the user can request that $\mathrm{Y}$ 
concepts are of semantic type pathologic function and that $\mathrm{Z}$ concepts are of semantic type pharmacologic substance. The last possibility for limiting the number of related concepts is by setting thresholds on the support and confidence measures of the association rules in steps 2 . and 3. of the algorithm. In fact, all of the filtering options can be interactively set alone or several of them in combination.

The goal of the ordering is to present best candidates first to make human review as easy as possible. Currently the default ordering is by the decreasing association rule confidence, but it is also possible to order by support or semantic type.

Implementation. Figure 1. shows the user interface of our discovery support system. The user starts a discovery session by searching for a starting concept $\mathrm{X}$, which is usually from his own research area. The concepts $\mathrm{Y}$ related to the starting concept are found by pressing the Find Related button and are presented in the Related Concepts1 frame. Before finding related concepts the user can specify limits and the order of the related concepts. Similarly the $\mathrm{Z}$ concepts related to the $\mathrm{Y}$ concepts are found and shown in the RELATED CONCEPTS2 frame. The frame RELATED CONCEPTS2 contains an important additional field designated as "Discovery?". This value of this field is YES if a relation (association) between the starting concept $\mathrm{X}$ and the current concept $\mathrm{Z}$ does not exist in the appropriate Medline segment and $N O$ if such a relation exists.

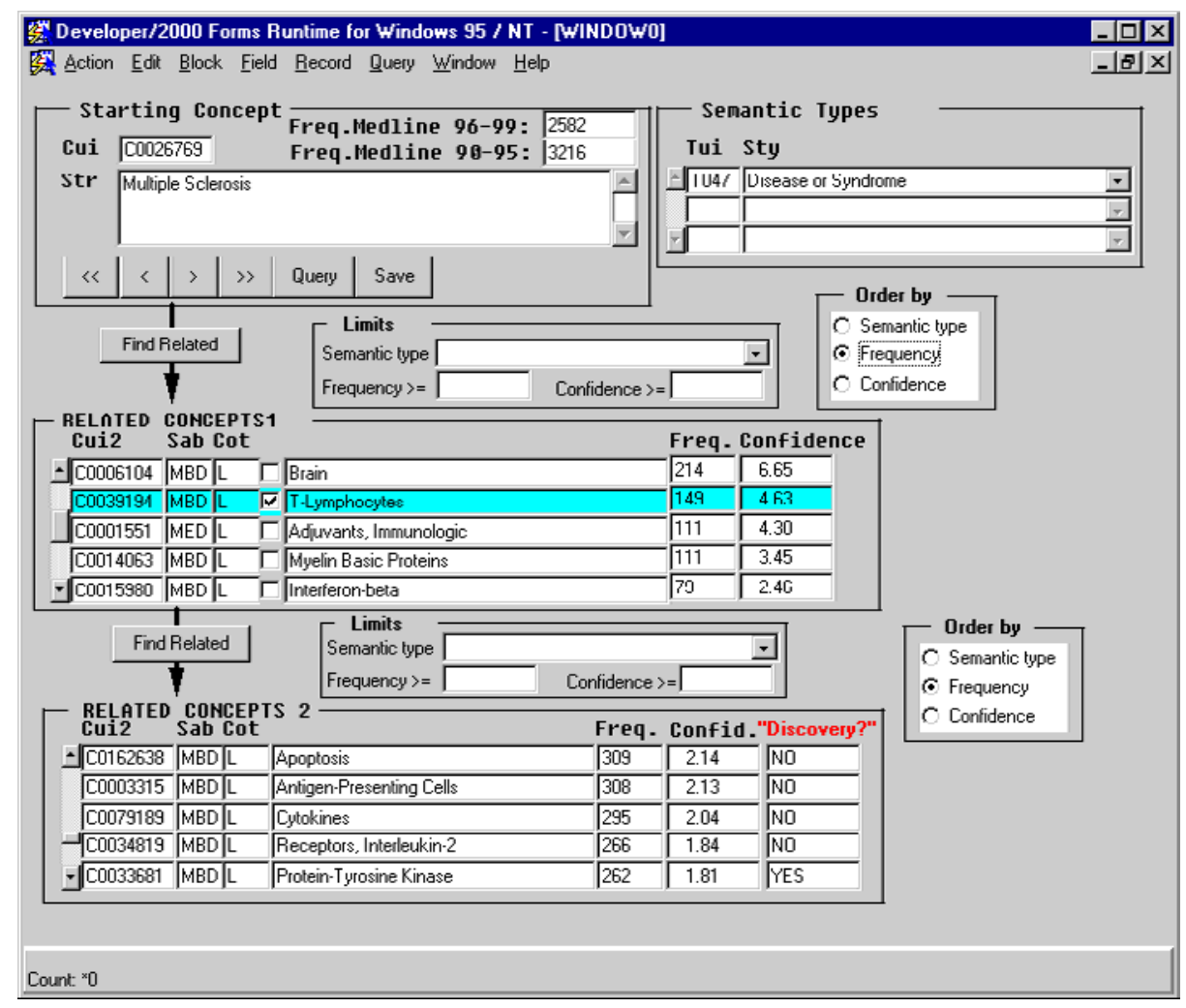

Fig. 1. The user interface of the interactive discovery support system. 


\section{Evaluation}

The ultimate proof of the system is to (help) discover medical discoveries that can be published in relevant medical journals. However, we have only managed to do a preliminary evaluation so far.

Medical Doctor. The main goal of the evaluation done by the medical doctor was to check if the $\mathrm{Y}$ concepts, which the system finds to be related to the starting concept $\mathrm{X}$, have medical sense. He selected as a starting concept multiple sclerosis (MS) which could be defined as a demyelinating disease of the central nervous system of putative autoimmune origin.

The first 5 concepts related (associated) with MS ordered by decreasing support of the association are: 1. MRI magnetic resonance imaging (diagnostics); 2. Brain (anatomical structure - organ involved); 3. Interferon (treatment).; 4. T-lymphocytes (pathogenesis); 5. Myelin basic protein (MBP)(pathogenesis).

Among the first 20 concepts there are 6 related to pathogenesis, 4 to treatment, 3 to diagnostic methods, 3 to symptoms, 2 to target organs-anatomical structures and 2 are related to general disease categories.

It could be estimated that the concepts found as related by the system are associated with the current main focus of medical endeavors in the field of MS which is still oriented to treatment and therefore to better understanding of pathogenesis.

Automatic. The goal of the automatic evaluation was to see how many of the potential discoveries made by the system at some point of time become realised at a later time. For us, a potential discovery is a relationship between two concepts proposed by our system, but not present in Medline at some point of time. We consider the potential discovery realised if the two concepts later appear together in a document in the Medline database. In other words, the goal of the evaluation was to see how good our system was in predicting what discoveries would be made in the future.

We approached this goal by first dividing the Medline database and the corresponding association rules into two segments according to the publication date of the documents stored: the older segment is from 1990 to 1995 and the newer segment is from 1996 to 1999 . We then analysed Multiple sclerosis $(M S)$, the same starting concept as in the medical doctor's evaluation.

MS appears in 2582 documents in the older segment. It is related to 1610 distinct concepts. When analysing the old segment, the system proposed 15617 concepts as potential discoveries. MS is related to 662 new concepts in the new segment that it was not related to in the old segment. Our system successfully predicted $95.5 \%(632$ out of 662) realised discoveries in the new segment. However, only 4\% (632 out of 15617) of the proposed potential discoveries got realised. It should be stressed that MS was not related to 15965 out of 17575 distinct concepts appearing in the older segment. The system proposed $97.8 \%$ of the concepts MS was not yet related to as potential discoveries. The conclusion is that without using limits on the strength of relationship the system is very successful at predicting future discoveries, but proposes far too many potential discoveries. Then we repeated the evaluation with two values for thresholds on the support level of the association rules. In one case the threshold was set to the average support of the associations between one concept and the others (AVGS) and in the other case it was set to $2 *$ AVGS. Only associations with support greater or equal to the threshold were taken into account. The number of 
proposed potential discoveries dropped from 15617 without thresholds to 6848 for AVGS and to 3151 for the $2 *$ AVGS threshold. The percent of successfully predicted realised discoveries dropped from $95.5 \%$ (632 of 662) without thresholds to 78.7\% (521 of 662) for AVGS and to 55.2\% (366 of 662) for $2 *$ AVGS. However, the ratio of realised to proposed potential discoveries improved from 4\% (632 out of 15617) without thresholds to 7.6\% (521 of 6848) for AVGS and to 11.6\% (366 of 3151) for $2 *$ AVGS. We conclude from this that with the use of proper thresholds the usability of the system is much better because a smaller number of better potential discoveries are generated.

\section{Discussion and Further Work}

The paper presented an interactive discovery support system for the field of medicine. For a given starting medical concept it discovers new, potentially meaningful relations with other concepts that have not been published in the medical literature before. The proposed relations should be evaluated and verified by a qualified medical professional.

As a measure of the relation between concepts we use association rules calculated from the Medline bibliographic database. As part of the preliminary evaluation of the system, a medical doctor confirmed that most of the relations between concepts found by association rules are meaningful. In the other part of the evaluation, which was done by automatic means, the system proved to be successful at predicting future discoveries. However, this came at the expense of generating a large number of potential discoveries that have to be judged and verified by the user of the system.

We have several ideas for improving the system. One of them is to develop a Web version of the system that will increase the number of users. More users means better chance for real medical discoveries. The preliminary evaluation showed that properly set thresholds are crucial for successful use of the system. Thus, we plan to work on setting good default values for the thresholds that can be changed by the user if necessary. Another important way to improve the system is to include additional information sources, such as molecular biology sequence databases.

\section{References}

1. Swanson, D.R.: Fish oil, Raynaud's syndrome, and undiscovered public knowledge. Perspect Biol Med. 1986 Autumn;30(1):7-18.

2. Swanson, D.R.: Online search for logically-related noninteractive medical literatures: a systematic trial-and-error strategy. J Am Soc Inf Sci. 1989 Sep;40(5):356-8.

3. Swanson, D.R., Smalheiser, N.R.: An interactive system for finding complementary literatures: a stimulus to scientific discovery. Artif. Intell. 91 (1997) 183-203.

4. U.S. National Library of Medicine. http://www.nlm.nih.gov/<30.04.2000

5. Humphreys, B.L., Lindberg, D.A.B., Schoolman, H.M., Barnett, G.O.: The Unified Medical Language System: an informatics research collaboration. JAMIA 1998;5(1):1-11.

6. Agrawal, R. et al: Fast discovery of association rules. In U. Fayyad et al, editors, Advances in Knowledge Discovery and Data Mining. MIT Press, Cambridge, MA. (1996) 